\title{
Optical, Magnetic and Dielectric Properties of Non-Liquid Crystalline Elastomers Doped with Magnetic Colloids
}

\author{
A.M. Figueiredo Neto, \\ Instituto de Física, Universidade de São Paulo, \\ Caixa Postal 66318, 05315-970, São Paulo, SP, Brazil \\ M.H. Godinho, \\ Faculdade de Ciências e Tecnologia e CENIMAT, Universidade Nova de Lisboa, \\ Quinta da Torre, P-2829-516 Caparica, Portugal \\ T. Toth-Katona, and P. Palffy-Muhoray \\ Liquid Crystal Institute, Kent State University, P.O.Box 5190, Kent, Ohio 44242-0001, USA
}

Received on 8 November, 2004; revised version received on 13 December, 2004

\begin{abstract}
Magnetic nanoparticles from magnetic colloidal suspensions were incorporated in the urethane/urea elastomer (PU/PBDO) by swelling fully crosslinked elastomer samples with a toluene and ferrofluid mixture. It is shown that ferrofluid grains can be efficiently incorporated into the matrix of elastomers. The dependence of the birefringence of both the pure and ferrofluid-doped elastomer samples on strain is linear. The ratio of birefringence to strain of the ferrofluid-doped samples is greater than that of the pure elastomer samples, indicating that ferrofluid grains are oriented by the strained polymer network. We propose that this strain-induced orientation is due to the shape anisotropy of the nanoparticles.
\end{abstract}

\section{Introduction}

Complex soft solids, such as liquid crystal elastomers [1] and ferrogels [2] are distinguished by their ready response to even modest stimuli. Because their sensitivity and responsivity makes them well suited for sensor and actuator applications, they are the subjects of considerable current interest.

Polypropylene oxide based urethane/urea elastomers have been widely used as pervaporation membranes. Their versatility allows the possibility of the design and realization of a wide variety of microstructures [3]. Recently, it was found that mechanical stress induces the formation of a variety of different patterns in these materials [4,5], and that the microstructures formed at the surfaces, revealed by atomic force microscopy (AFM), can be tuned by varying the magnitude and direction of the externally applied mechanical field [6].

Ferrofluids, or magnetic fluids [7-9], are colloidal suspensions of small magnetic grains, with typical dimensions of the order $10 \mathrm{~nm}$, dispersed in a liquid carrier. Ferrofluids, in the absence of external fields, are optically isotropic, and possess large magnetic susceptibility. However, fields which orient the grains, such as magnetic [10] and surface $[11,12]$ fields, induce birefringence in ferrofluids. Two different types of ferrofluids are available. Surfacted and ionic ferrofluids differ in the physical processes used to avoid agglomeration of the grains. Surfacted ferrofluids $(S F F)$ consist of grains, usually magnetite $\left(\mathrm{Fe}_{3} \mathrm{O}_{4}\right)$, coated with surfactants, consisting of amphiphilic molecules (e.g., oleic acid and aerosol sodium di-2 ethylhexyl-sulfosuccinate) to prevent their aggregation. Steric repulsion between grains acts as a physical barrier [13] which keeps the grains in the solution, stabilizing the colloid. If the grains are dispersed in a polar medium (e.g., water), two layers of surfactant are needed to form an external hydrophilic layer. Surfactant molecules with polar heads can be cationic, anionic or nonionic. On the other hand, when grains are dispersed in a nonpolar medium (e.g., light mineral oil, toluene, octane, hexane, cyclohexane), a single surfactation is enough, with the polar head of the surfactant attached to the surface of the grains and the carbonic chain in contact with the carrier fluid. In the case of ionic ferrofluids [14], repulsive interaction is maintained by electrostatic forces.

Elastomers swollen with ferrofluid particles, known as ferrogels, have received considerable attention $[15,16]$. They are usually formed either by precipitation of the magnetic particles in the polymer material before, during or after the cross-linking process [17], or by mixing the polymer solution and the fully formed magnetic sol together, and subsequently polymerizing [18]. These materials present remarkable magnetoelastic and magnetostrictive properties. Ferrogels present giant elastic response when subjected to (even modest) magnetic fields. This property, in particular, has not only interest from the fundamental point of view (coupling between elasticity and magnetic torque) but also allows interesting technological applications for this complex material.

Another possible way to introduce magnetic particles in 
the elastomer is to swell the fully cross-linked material with a solvent containing ferrofluid particles, and then remove the solvent. The magnetic particles are expected to diffuse into the elastomer together with the solvent, and then remain trapped in the network once the solvent is removed by evaporation during drying.

In this paper, we describe the process of producing ferrogels in this way, and discuss characterization of the material and its response by using atomic force microscopy (AFM) and electric permittivity and magnetic susceptibility measurements. Optical birefringence measurements as function of strain are used on both pure and doped samples to study strain induced orientational order. Our main interest is to investigate the orientational order produced in these materials as consequence of mechanical strain.

\section{Experimental}

\subsection{Sample preparation}

The urethane/urea elastomer was prepared by the reaction of the end groups of the tri-functional polypropylene oxidebased isocyanate prepolymer (PU) (Fig.1a) with molecular weight of 3500, and polybutadiene diol (PBDO) (Fig.1b) with molecular weight of 2800 , catalyzed by dibutyltin dilauryate (DBTDL).

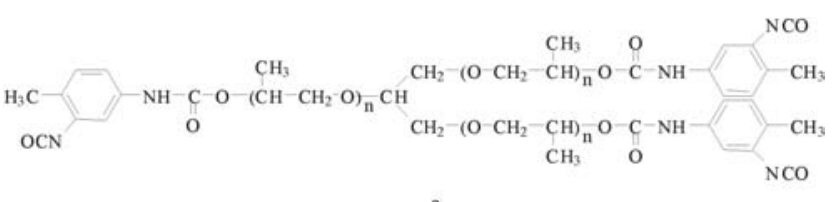

$$
\mathrm{OH}\left(\mathrm{CH}_{2}-\mathrm{CH}=\mathrm{CH}-\mathrm{CH}_{2}\right)_{\mathrm{n}} \mathrm{OH}
$$

Figure 1. Chemical structures of: a) tri-functional polypropylene oxide-based isocyanate prepolymer (PU), $n=20 ; b)$ polybutadiene diol (PBDO), $\mathrm{n}=50$.

The PU and PBDO prepolymers, in a molar ratio of $3: 2$ (PU: PBDO), were dissolved in toluene, under nitrogen atmosphere according to the procedure described in ref. [19]. Following a 30 min period during which reaction was allowed to proceed, the solution was simultaneously cast and sheared by moving a calibrated Gardner casting knife with speed of $5 \mathrm{~mm} / \mathrm{s}$, onto a coated glass plate, at room temperature. After curing in an oven at $75^{\circ} \mathrm{C}$ for $3.5 \mathrm{~h}$, the elastomer was exposed to air. The reaction of water from atmospheric moisture with excess of isocyanate groups in PU yields urea linkages. The colorless elastomer film, approximately $150 \mu \mathrm{m}$ thick, was removed from the glass substrate, and was studied subsequently as a free standing film.

\subsection{Ferrofluid doping}

The $S F F$ used to dope the elastomer was obtained from Ferrotec Corp. [20]. The ferrofluid is from the EMG 911 series, consisting of magnetite $\left(\mathrm{Fe}_{3} \mathrm{O}_{4}\right)$ grains in a mineral oil carrier. The typical grain diameter is $10 \mathrm{~nm}$, the saturation magnetization is $11 \mathrm{mT}$, and the viscosity is below $5 \mathrm{mPa} \cdot \mathrm{s}$. The volume fraction of magnetite in the ferrofluid is between 3 to $15 \%$. Although ferrofluid grains are typically referred to as spheres, in fact, they have irregular, somewhat elongated shapes, resembling potatoes. This shape anisotropy can be seen in AFM experiments on dried ferrofluids [21], and is responsible for the ability of ferrofluids to mechanically orient liquid crystals [22]. In addition to this grain shape anisotropy, small scale aggregation usually occurs in ferrofluids, resulting in the formation dimers and trimers [23], which are again elongated in shape.

A mixture of ferrofluid in toluene with volume ratio $4: 1$ was prepared to swell the elastomer and to allow the magnetic grains to diffuse into the sample. No agglomeration of magnetic grains was observed in the mixture. The solution was stable, remaining homogeneous during the 6 days of monitoring. Elastomer samples of varying sizes were cut from the $150 \mu \mathrm{m}$ thick sheets. To dope with ferrofluid, elastomer samples were immersed in the toluene-ferrofluid solution and were allowed to stand undisturbed for 8 hours. After rinsing in toluene to remove any excess ferrofluid from the surfaces, samples were placed in a vacuum oven for drying for 8 hours. All of the above processes were carried out at room temperature. After drying, the elastomer samples, which were initially transparent, acquire a brown color due to the presence of magnetite grains. The dimensions of the sample after drying remain the same (within our accuracy: length and width measured with a paquimeter and thickness with a micrometer) as the previous undoped sample.

\subsection{Atomic force microscopy}

Atomic force microscopy (AFM) was carried out on freshly cut surfaces of pure (undoped) and doped (ferrogel) samples. Fig. 2 shows the AFM pictures of undoped and ferrofluid doped elastomer. The surfaces studied were edges of the elastomer film, formed by cutting perpendicular to the largest sample surfaces, in the $y z$ plane of the laboratory frame (see Fig. 2a).

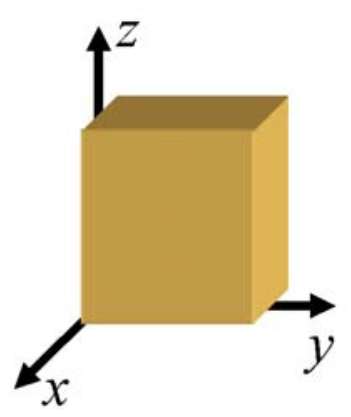

Figure 2a. Orientation of elastomer sheet in the laboratory frame. 


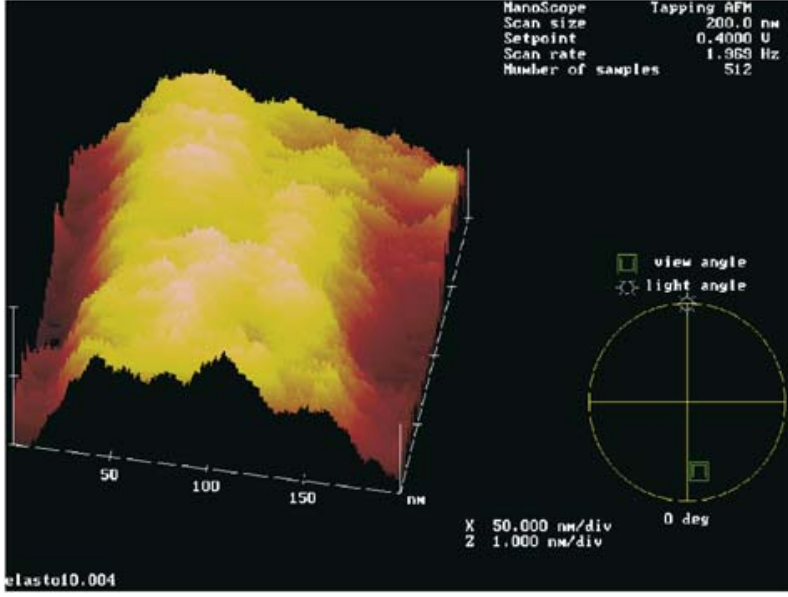

Figure $2 b$. Atomic force micrographs of elastomer samples in tapping mode: undoped elastomer, cross section in the $x y$ plane.

Figure $2 \mathrm{~b}$ shows the typical surface structure of the undoped elastomer sample on the $200 \mathrm{~nm}$ scanning scale. The pattern is rather featureless, indicating an essentially flat surface. On the other hand, the pattern of the ferrofluid doped sample (Fig. 2c) presents a different topography, characterized by peaks and valleys. The peaks, with typical widths in the range of $\sim 20$ to $\sim 50 \mathrm{~nm}$, correspond to the ferrofluid grains and small-scale aggregates incorporated in the elastomer matrix.

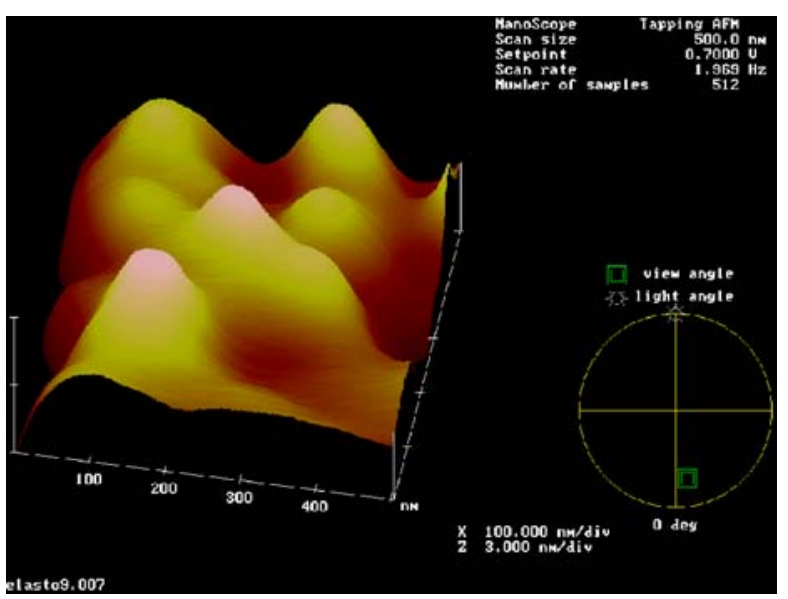

Figure 2c. Atomic force micrographs of elastomer samples in tapping mode: ferrofluid doped elastomer, cross section in the $x y$ plane.

\subsection{Optical measurements}

In order to study strain induced order, we determined the difference in the optical phase shift for light polarized parallel and perpendicular to the stretching direction. The experimental geometry is shown in Fig. 3.

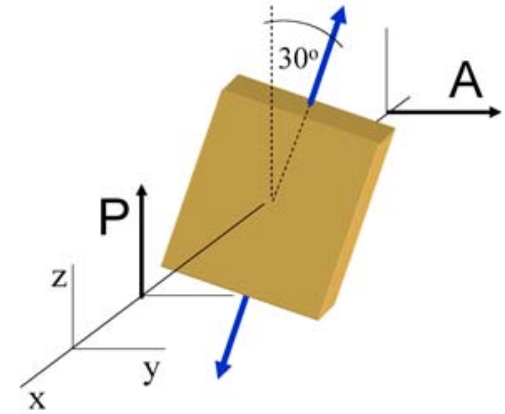

Figure 3. Sketch of the optical setup. The laser beam propagates along the $x$ axis, $\mathrm{P}$ and $\mathrm{A}$ are the directions of the axes of the polarizer and analyzer. The sample is stretched in the direction indicated by the arrows.

A HeNe laser beam $(\lambda=632.8 \mathrm{~nm})$, polarized along the $z$ direction after passing through polarizer $P$, propagates along the $x$ axis and is normally incident on a sample, which is stretched in the direction indicated by the arrow. The transmitted light passes through analyzer $A$ parallel to the $y$ axis and its intensity is measured by a photodiode. The sample can be stretched along the direction $\sim 30^{\circ}$ from the $z$ axis. Without the stretching, the sample is isotropic and the measured intensity of transmitted light is zero. As the sample is stretched, it becomes birefringent. We have measured the transmitted intensity as a function of sample strain. The maximum sample elongation was $\Delta l=l-l_{o} \simeq 1 \mathrm{~mm}$; typical sample dimensions were $100 \mu \mathrm{m} \times 4 \mathrm{~mm} \times 7 \mathrm{~mm}$, and elongation was along the direction of longest sample dimension $l_{o}=7 \mathrm{~mm}$.

\subsection{Paramagnetic susceptibility measure- ments}

A ferrofluid doped sample with the same dimensions as above was placed in a magnetic field gradient, and the force on the sample was measured using an Entran force sensor. The ferrogel sample, suspended from the arm of the Entran sensor by a light thread, was placed near the poles of an electromagnet, where the field variation measured with a Hall probe, was found to be $\sim 1 \mathrm{kG} / \mathrm{cm}$ in the $x$ direction, as shown in Fig. 4.

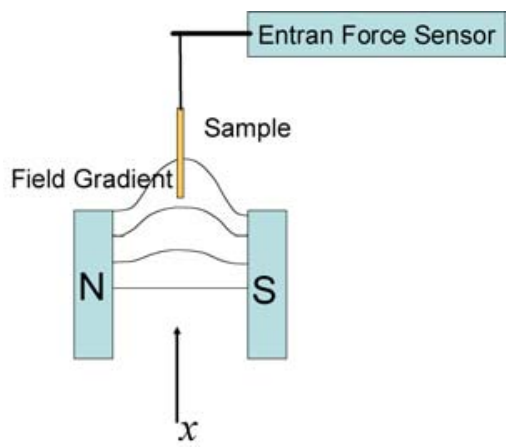

Figure 4. Sketch of setup used to measure the magnetic force on the ferrofluid doped elastomer sample. 
The Entran force sensor uses a piezoelectric crystal whose amplified output voltage is proportional to the force on the sensor arm. The output of the amplifier was measured with an oscilloscope.

\subsection{Electric permittivity $(\varepsilon)$ measurements}

We determined the dielectric permittivity of both pure and ferrofluid doped elastomer samples via capacitance measurements. We constructed parallel plate capacitors by placing our samples between glass plates coated with indium tin oxide. The capacitance was measured at 1 and $5 \mathrm{kHz}$ using a GenRad 1615 Capacitance Bridge, with an external Hewlett-Packard Signal Generator and a Stanford Research lock-in amplifier as detector. The relative dielectric constant $\varepsilon_{r}$ is obtained from the capacitance via

$$
\varepsilon_{e}=\frac{C d}{\varepsilon_{o} A}
$$

where $d$ and $A$ are the sample thickness and area, respectively.

\section{Results and discussion}

\subsection{Swelling behavior}

The urethane/urea elastomer was swelled with six different pure solvents: chloroform, toluene, acetone, isopropanol, ethanol and methanol. Samples were placed between two glass plates separated by $400 \mu \mathrm{m}$ spacers to avoid bending of the elastomer during the swelling process. Solvents were introduced between the glass plates to swell samples, and sample lengths (along the $z$-axis) and widths (along the $y$ axis) were measured as function of time. Typical sample dimensions are: $100 \mu \mathrm{m} \times 3 \mathrm{~mm} \times 3 \mathrm{~mm}$. We define the percent relative expansion, a function of time, as

$$
\frac{\Delta l(t)}{l_{o}}=\frac{l(t)-l_{o}}{l_{o}} \times 100
$$

where $l(t)$ is the length of the swelled sample, measured at the time $t$, and $l_{o}$ is the length of the unswelled sample. Figures 5a and 5b show typical results of the swelling process, with chloroform and methanol, respectively. The errors were calculated using classical error-propagation procedures.

Table 1 presents a summary of the characteristic swelling times (obtained fitting an exponential function to the different percent relative expansion curves) and the relative expansion.

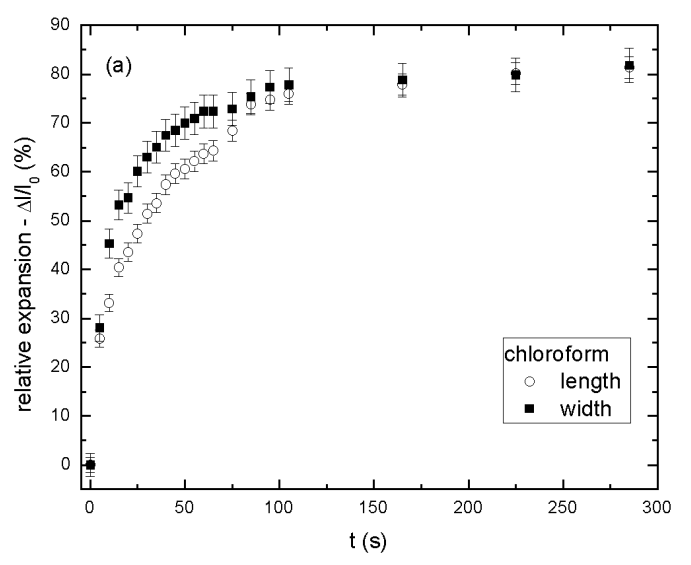

Figure 5a. Relative expansion $\left(\Delta l / l_{o}\right)$ as function of time in the swelling experiment: ( $($ ) length and (ם) width of the sample in the solvent chloroform.

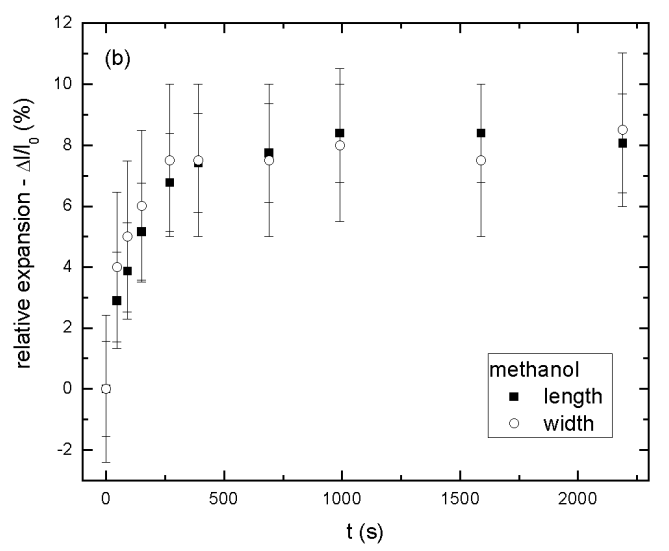

Figure 5b. Relative expansion $\left(\Delta l / l_{o}\right)$ as function of time in the swelling experiment: (ם) length and (o) width of the sample in the solvent methanol.

TABLE 1. Characteristic swelling times and relative expansion of the PU/PBDO elastomer with different solvents.

\begin{tabular}{|c|c|c|c|c|}
\hline & \multicolumn{2}{|c|}{ characteristic times $(s)$} & \multicolumn{2}{c|}{ relative expansion (\%) } \\
\hline solvent & length & width & length & width \\
\hline chloroform & $35.5 \pm 3.2$ & $17 \pm 2$ & 80 & 80 \\
\hline toluene & $23.3 \pm 1.3$ & $20.2 \pm 1.6$ & 75 & 70 \\
\hline acetone & $31.1 \pm 1.8$ & $20.2 \pm 2.3$ & 25 & 25 \\
\hline isopropanol & $182 \pm 48$ & $86 \pm 15$ & 12 & 12 \\
\hline ethanol & $151 \pm 13$ & $156 \pm 16$ & 12 & 12 \\
\hline methanol & $148 \pm 14$ & $85.0 \pm 0.5$ & 8 & 8 \\
\hline
\end{tabular}

These results indicate that the swelling is nearly isotropic in the $y z$ plane. Concerning the different characteristic swelling times along the width and and length of the 
sample, it seems that the solid film preparation and casting, namely the solvent evaporation associated with the shear rate of the casting knife, induced an orientational order in the cross-linked solid film that gives rise to the anisotropic behavior observed [19]. Chloroform and toluene were the most efficient solvents, with the smallest characteristic times and largest relative expansions. Toluene was therefore chosen as the solvent for the ferrofluid doping procedure. Toluene is the solvent used to prepare the elastomer (both pre-polymers have a great affinity for this solvent) this was one of the reasons to choose the toluene for the ferrofluid doping procedure.

\subsection{Ferrofluid doped samples}

Magnetic susceptibility measurements were used to determine the density of grains in the sample. A sample with dimensions of $100 \mu \mathrm{m} \times 4 \mathrm{~mm} \times 7 \mathrm{~mm}$, placed in a field gradient of $1 \mathrm{kG} / \mathrm{cm}$ experienced a force of $\sim 4 \times 10^{-4} \mathrm{~N}$. Assuming [24] that the typical value for the magnetic moment of a grain is $10^{4} \mu_{B}$, where $\mu_{B}$ is the Bohr magneton, the calculated number density of grains in the sample is $\sim 1.5 \times 10^{17} \mathrm{~cm}^{-3}$. This corresponds to a concentration of $\sim 10 \%$ (by volume) of magnetic grains, which is in reasonable agreement with the topographs indicated by AFM in Fig. 2c.

An interesting observation obtained with a freshly prepared ferrofluid doped elastomer sample (after drying) is that the sample is similarly attracted by small permanent magnets with fields of $\sim 100 \mathrm{G}$.

Using the optical setup described in the experimental section, we measured the transmittance $T$ of the sample as a function of the stretching $\Delta l$. The absolute value of the optical phase shift $|\Delta \phi|$ can be calculated from the values of the normalized transmittance $T_{N}=T / T_{M}$, where $T_{M}$ is the maximum transmittance, as

$$
|\Delta \phi|=2 \arcsin \left(\sqrt{T_{N}}\right)
$$

Figure 6 shows $|\Delta \phi|$ as a function of $\Delta l$ for a pure elastomer sample, and for an elastomer swelled with toluene and ferrofluid and subsequently dried.

The error, calculated from the experimental transmittance measurements, is about $\pm 2.5 \%$ of the $|\Delta \phi|$ value, for each point of Fig. 6. For both pure and ferrofluid doped samples, the induced phase shift, and hence the birefringence, are proportional to the strain and hence to the stress, as expected from the stress-optic law [25]. The results clearly demonstrate the influence of the presence of magnetic grains in the elastomeric matrix, which increases the stress-induced optical phase shift compared to the values obtained in undoped samples. We note that although it is recognized in the literature $[2,16]$ that the stress in the network is coupled to the magnetization, the origins of this coupling have not been identified. We propose that the coupling, and this stress-induced optical phase shift, originates in the mechanical orientation of anisometric grains (and small scale aggregates of grains). In this picture, the long axes of grains and aggregates become parallel to the direction of elongation of the elastomer in order to reduce the free energy of the deformed elastomer. It is interesting to note that the shape anisotropy of both grains and small scale aggregates on the average, has quadrupolar symmetry, while the corresponding magnetic moment is dipolar. Ferrofluids therefore combine dipolar and quadrupolar symmetry when incorporated into the elastomer matrix.

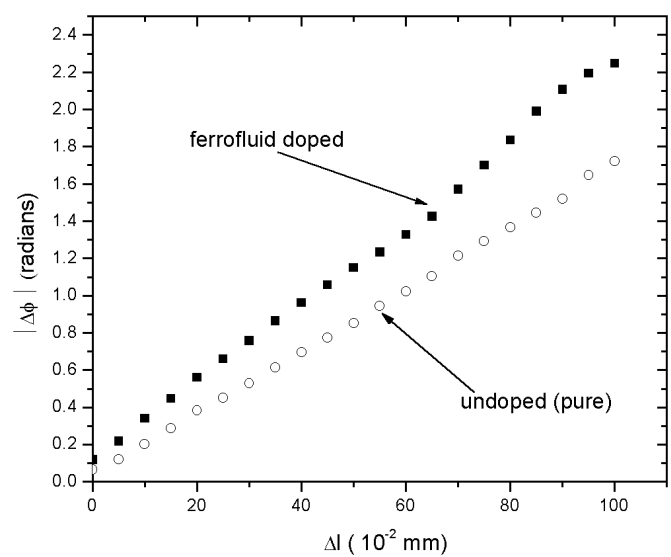

Figure 6. Absolute value of the strain-induced optical phase shift $(|\Delta \phi|)$ as a function of the sample stretch $(\Delta l)$ at $\sim 25^{\circ} C$ for pure and ferrofluid doped elastomer.

We have estimated the absolute value of the optical anisotropy [23] of our ferrofluid doped samples assuming perfectly aligned ferrofluid particles. The particles are considered to be prolate ellipsoids particles with axes of length $2 a$ and $2 b,(a>b)$, and aspect ratio $b / a=0.93969$, which corresponds to a shape anisotropy of about $5 \%$. In this framework, the ellipsoidal ferrofluid grain has different depolarizing factors for light polarized parallel or perpendicular to the infinite-fold symmetry axis of the grain [26]. This shape anisotropy imposes to the grain a form birefringence $[27,28]$, which is now evaluated in our case. The volume fraction of particles is $10 \%$, and we obtain the absolute value of the birefringence at saturation [23] $|\Delta n| \simeq 8 \times 10^{-4}$. In our experiment above, we note that the maximum phase shift increase due to the presence of the ferrofluid particles is $0.4 \mathrm{rad}$, and for $d_{o}=100 \mu \mathrm{m}$ thick samples (at this point we have assumed that the stretching does not significantly modify the sample thickness) and $\lambda=632.8 \mathrm{~nm}$, $\left|\Delta n_{\text {exp }}\right|=4 \times 10^{-4}$. There is therefore reasonable agreement between theoretical estimates and experimental observations. Let us discuss now the hypothesis made about the invariance of the sample thickness $(d)$ upon stretching in this evaluation. In a more realistic picture,

$$
\frac{d}{d_{o}} \approx \frac{l_{o}}{l_{\max }}
$$

where $l_{\max }$ is the maximum value of $l$. Estimating Eq. 2 one has $d / d_{o} \sim 0.88$, which increases by about $15 \%$ our estimate of $\left|\Delta n_{\exp }\right|$. 
In all the cases studied, a small hysteresis was observed in a cycle increasing and after decreasing $\Delta l$. We have also verified that the ferrofluid doped sample, not stretched but subjected to a magnetic field up to $13 k G$, does not exhibit a field-induced phase shift. At this relatively large field, it is expected that the magnetic moments of the grains align parallel to the field in a Néel-type rotation of the magnetization [29], without physical rotation of the grains, which remain fixed in the elastomer structure.

Capacitance measurements were carried out to determine the dielectric constant of elastomer samples, both undoped and doped with ferrofluids. The results were independent of frequency, between 1 and $5 \mathrm{kHz}$. The dielectric constants of the doped and undoped samples were found to be 14.1 and 10.5 , respectively. We can make a crude estimate of the expected value of the dielectric constant $\varepsilon_{e}$ of the elastomer doped with ferrofluid using simple effective medium theory. The effective dielectric constant $\varepsilon_{e}$ of a mixture consisting of volume fraction $(1-p)$ of component $U$ with dielectric constant $\varepsilon_{U}$ and volume fraction $p$ of component $F$ with dielectric constant $\varepsilon_{F}$ is [30]

$$
(1-p) \frac{\varepsilon_{U}-\varepsilon_{e}}{\varepsilon_{U}+2 \varepsilon_{e}}+p \frac{\varepsilon_{F}-\varepsilon_{e}}{\varepsilon_{F}+2 \varepsilon_{e}}=0 .
$$

Assuming that at low frequencies the dielectric constant of the metal magnetic particles $\left|\varepsilon_{F}\right|>>\varepsilon_{e}$, we get, using $p=0.1$,

$$
\varepsilon_{e}=\frac{\varepsilon_{U}}{3 p-2}=15.0
$$

in reasonable agreement with the measured value of 14.1.

\section{Conclusion}

We have shown that ferrofluid grains can be efficiently incorporated into the matrix of PU/PBDO elastomers by swelling fully crosslinked elastomer samples with a toluene and ferrofluid mixture. The magnetic particles remain embedded in the elastomer after the solvent is removed. The presence of ferrofluid particles is evidenced by AMF, magnetic susceptibility and dielectric measurements. The dependence of birefringence of both pure and ferrofluid doped elastomer (ferrogel) samples on strain is linear, as expected. The ratio of birefringence to strain of the ferrogel samples is greater than those of the pure elastomers, indicating that ferrofluid grains are oriented by the strained polymer network. Although the mechanism of the coupling between the orientation of grains and clusters and the network has not yet been clearly identified, we propose it is due to shape anisotropy.

\section{Acknowledgement}

We acknowledge the Fundação de Amparo à Pesquisa do Estado de São Paulo (FAPESP) and the U.S. National Science Foundation under grant DMR - 0132611 for financial support. Ms. Liou Qiu and Dr. O. D. Lavrentovich, from the characterization facilities of the LCI, KSU are acknowledged for the AFM measurements.

\section{References}

[1] M. Warner and E.M. Terentjev, Liquid Crystal Elastomers, Oxford University Press, Oxford (2003).
[2] M. Zrínyi, L. Barsi, and A. Büki, J. Chem Phys. 104, 8750 (1996).

[3] C.-T. Zhao, M.N. de Pinho, Polymer 40, 6089 (1999).

[4] M.H. Godinho, J.L. Figueirinhas, C.-T. Zhao, and M.N. de Pinho, Macromolecules 33, 7675 (2000).

[5] M.H. Godinho, J.L. Figueirinhas, C.-T. Zhao, and M.N. de Pinho, Mol. Cryst. Liq. Cryst. 365, 447 (2001).

[6] M.H. Godinho, L.V. Melo, and P. Brogueira, Mat. Sci. Eng. C-Bio S 23, 919 (2003).

[7] S. S. Papell, US Patent 3.215.572, October $9^{\text {th }}$ (1963).

[8] R. E. Rosensweig and R. Kaiser, NTIS Rep. No. NASW-1219 (1967).

[9] R. E. Rosensweig, Ferrohydrodynamics Cambridge Univ. Press, Cambridge, (1985).

[10] Magnetic Fluids and Applications Handbook, edited by B. Berkovski and V. Bashtovoy, Begell House, Wallingford (1996).

[11] C. Matuo, A. Bourdon, A. Bee, and A. M. Figueiredo Neto, Phys. Rev. E 56, R1310 (1997).

[12] M. Magalhães, A.M. Figueiredo Neto, A. Bee, and A. Bourdon, J. Chem. Phys. 113, 10246 (2000).

[13] S. W. Charles and J. Popplewell, in Ferromagnetic Material, edited by E. P. Wohfarth, North-Holland Publishing Company, Vol. 2, Amsterdam, (1980).

[14] R. Massart, C. R. Acad. Sci. Paris 291C, 1 (1980).

[15] M. Zrínyi, L. Barsi, and D. Szabó, J. Chem Phys. 106, 5685 (1997).

[16] T. Mitsumata, K. Ikeda, J. P. Gong, Y. Osada, D. Szabó, and M. Zrínyi, J. App. Phys. 85, 8451 (1999).

[17] J.E. Mark, Brit. Polym. J. 17, 144 (1985).

[18] W. Haas, M. Zrínyi, H-G. Killian, and B. Heise, Colloid. Polym. Sci. 271, 1024 (1993).

[19] A.C. Trindade, M.H. Godinho, and J.L. Figueirinhas, Polymer 45, 5551 (2004).

[20] http://www.ferrotec.com

[21] C. Quilliet, P. Fabre, and V. Cabuil, J. Phys. Chem. 97, 287 (1993).

[22] F. Brochard and P. G. de Gennes, J. Physique 31, 691 (1970).

[23] M. F. da Silva and A. M. Figueiredo Neto, Phys. Rev. E 48, 4483 (1993).

[24] M.H. Sousa, F.A. Tourinho, J. Depeyrot, G.J. da Silva, and M.C.F.L. Lara, J. Phys. Chem. B 105, 1168 (2001).

[25] M. Doi, Introduction to Polymer Physics, Clarendon Press (1996).

[26] J. A. Osborn, Phys. Rev. 67, 351 (1945).

[27] E. Frederick and C. Houssier, Electric Dichroism and Electric Birefringence, Oxford: Oxford University Press (1973).

[28] H. W. Davies and J. P. Llewellyn, J. Phys. D: Appl. Phys. 12, 1357 (1979).

[29] L. Néel, Ann. Geophys. 5, 99 (1949); C.R. Acad. Sci. 228, 664 (1949).

[30] R. Landauer, J. Appl. Phys. 23, 779 (1952). 